\title{
Effects of imperfect experimental conditions on stress waves in SHPB experiments
}

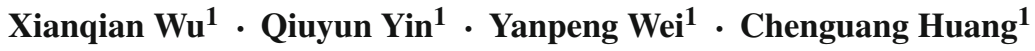

Received: 5 September 2014 / Revised: 14 November 2014 / Accepted: 12 January 2015 / Published online: 14 August 2015

(C) The Chinese Society of Theoretical and Applied Mechanics; Institute of Mechanics, Chinese Academy of Sciences and Springer-Verlag Berlin Heidelberg 2015

\begin{abstract}
Experimental and numerical simulations were undertaken to estimate the effects of imperfect conditions on stress waves in split Hopkinson pressure bar (SHPB) experiments. The photonic Doppler velocimetry (PDV) measurement results show that the rise and fall times of an incident wave increases with an increasing inclination angle; also, the fluctuations of the incident wave disappear gradually with the increase of inclination angle. The following characteristics for various defects in the SHPB were obtained by numerical simulation: (1) the influence of a curved bar was negligible; (2) misalignment modestly affects the fluctuation characteristics, and bending waves were generated at this condition; (3) inclination and indentation of the impact endsurface had a great impact on the incident waves, and both of them increase the rise time of the incident wave by increasing the degree of defects. In view of the results, misalignment, inclination, and indentation in SHPB experiments should be minimized.
\end{abstract}

Keywords Imperfect experimental conditions - SHPB experiments $\cdot$ Stress waves $\cdot$ PDV measurement $\cdot$ Numerical simulation

\section{Introduction}

The split Hopkinson pressure bar (SHPB), which was first introduced by Hopkinson [1] in 1914 and further developed

\footnotetext{
Xianqian $\mathrm{Wu}$

wuxianqian@imech.ac.cn

1 Key Laboratory of Mechanics in Fluid Solid Coupling Systems, Institute of Mechanics, Chinese Academy of Sciences, Beijing 100190, China
}

by Kolsky [2], Davies and Hunter [3], as well as others [4-7], is an effective method to characterize the dynamic behavior of materials at intermediate strain rates. The SHPB has been widely used to investigate the dynamic mechanical properties of materials under uniaxial compression $[5,6,8$ 11]. The SHPB apparatus comprises three elastic bars that are the striker, the incident bar, and the transmitted bar. A specimen is sandwiched coaxially between the incident bar and the transmitted bar. Once the striker impacts the incident bar, a stress pulse is generated and propagates through the incident bar towards the specimen. When the incident stress wave arrives at the incident bar/specimen interface, a reflective wave is generated and propagates backwards along the incident bar due to impedance mismatch, and the rest transmits through the specimen to the transmitted bar. From the incident, the reflected, and the transmitted strain signals, the stress-strain relationships of the specimen at various strain rates can be obtained. The characteristics of the incident strain pulse, such as the rise time, affect the measurements of the materials' stress-strain relationships greatly, especially for brittle materials such as concrete, ceramic, and cryogenic ice $[4,5,12]$. In addition, the stress equilibrium condition along the specimen thickness is required in SHPB experiments [13], which could also be facilitated by a ramp incident wave with a relatively long rise time, especially for brittle materials. Many researchers focus on the effects of the end-friction, the end-face indentation, the processing methodology of measured data, and some new experimental methods to get more accurate constitutive laws accounting for the accurate dispersion correction and the exact delay setting $[9,10,14-19]$. However, the imperfect experimental conditions, e.g. defects, of SHPB experiments, such as the inclination of the striker/incident contact surfaces, the curved bars, or the misalignment of bars and 
the end-surface indentation, which may affect the measurement results and thereby decrease experimental accuracy, are rarely quantitatively studied in the open literature. The investigation of the defects in SHPB experiments not only provides insight to the influence of the imperfect condition effects, but also aids in understanding the experimental results.

In the present research, the effects of imperfect conditions on the incident stress waves of the SHPB were studied by experiments and numerical simulation. Four kinds of defects that are the curved bar, the inclination of the impact end-surface, the misalignment between the striker and the incident bar and the indentation of the impact end-surface, were investigated. Firstly, the effects of the inclination of the incident bar with respect to the striker were investigated by SHPB experiments by measuring the strain pulses and endsurface velocities of the incident bar with strain gauges and a photonic Doppler velocimetry (PDV), respectively. Note that the effects of the curved bar and the inclination of the impact end-surface were coupled. The PDV system, which is based on the principle of Doppler interference, has been rapidly gaining popularity as a powerful diagnostic method for tracking particle velocities on short time scales in shock dynamics experiments since its invention [20-22]. It has excellent functions on low velocity and multiple simultaneous velocity measurements. The measured results show that the designed imperfect experimental conditions have great impacts on the incident waves. Then, to decouple the effects of the curved bar and the inclination of the impact end-surface, these influences were studied individually by numerical simulation. The simulated results indicate that the effects of the curved bar are negligible, whereas the inclination of the impact endsurface affects the pulse shape significantly, including the generation of obvious bending waves and increasing the rise time. Finally, the effects of the misalignment between the striker and the incident bar as well as the indentation of the impact end-surface were also simulated. The results show that the fluctuations induced by the lateral inertia effect the decrease slightly and the bending waves increase slightly with increasing the degree of the misalignment. In addition, the pulse shapes were affected significantly by the indentation of the impact end-surface. The rise and the fall times increase quickly and the dispersion effects disappear gradually with increasing the deflection of the impact end-surface.

The article is organized as follows. In Sect. 2, the SHPB experiments for the different inclination angle of the incident bar were described. In Sect. 3, the schematic of numerical simulations were designed to analyze the influence of the four kinds of imperfect experimental conditions. In Sect. 4, the experimental results and the simulated results were given. The effects of the imperfect experimental conditions on the incident waves were investigated and discussed.

\section{Experiments}

A simplified SHPB apparatus equipped with a PDV system was used to investigate the influence of the imperfect experimental conditions. The schematic diagram of the experimental is depicted as Fig. 1, in which the inclination of the incident bar is designed. Only the striker and the incident bar were used in this study to simplify the problem. C1045 steel is used for both the striker and the incident bar. The geometrical and material parameters for the SHPB system are listed in Table 1 [23]. A thin layer of lubricant was applied on the impact end-surface of the incident bar to minimize the effect of friction. The construction and the measuring principle for the PDV system is described in detail elsewhere $[24,25]$. The incident velocity of the striker was measured by a high-precision laser velocimetry and kept almost constant by using the same chamber pressure in each experiment. A pair of strain gauges were strategically cemented on the middle of the incident bar (denoted as upper and lower strain gauges) and used in combination with a Wheatstone bridge circuit connected with a differential amplifier (Tektronix $5 \mathrm{~A} 22 \mathrm{~N}$ ) and a digital oscilloscope (Lecoy WaveMaster $808 \mathrm{Zi}$ ) to record the stress pulses during the tests. The probe of the PDV system with a work distance of $15 \mathrm{~mm}$ was fixed opposite to the center of the end-surface of the incident bar to capture the particle velocity. Teflon tape was wrapped around the incident bar near the bearing as depicted in Fig. 1 to protect the probe. The PDV measurement was triggered by the rising edge of the strain signals of the incident bar. Three angles of inclination $\beta=0^{\circ}, 1^{\circ}, 2^{\circ}$ of the incident bar were designed by adjusting the height of the right end of the incident bar in experiments. The incident waves of the incident bar for various angles of inclination $\beta$ were investigated by the strain gauges and the PDV system.

\section{Numerical simulation}

\subsection{Schematic of simulation}

In Sect. 2, the experiments were conducted to investigate the influence of the inclination of the incident bar, in which

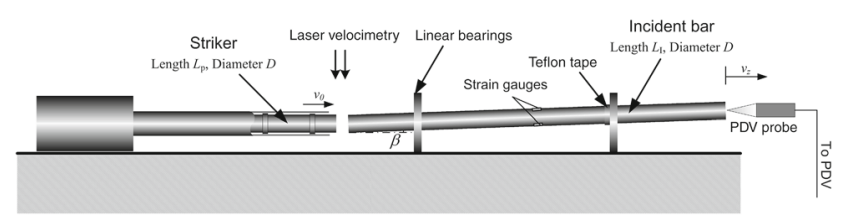

Fig. 1 Schematic diagram of experiments. Only the striker and the incident bar were used. Different inclination angle of the incident bar with respect to the striker was designed 


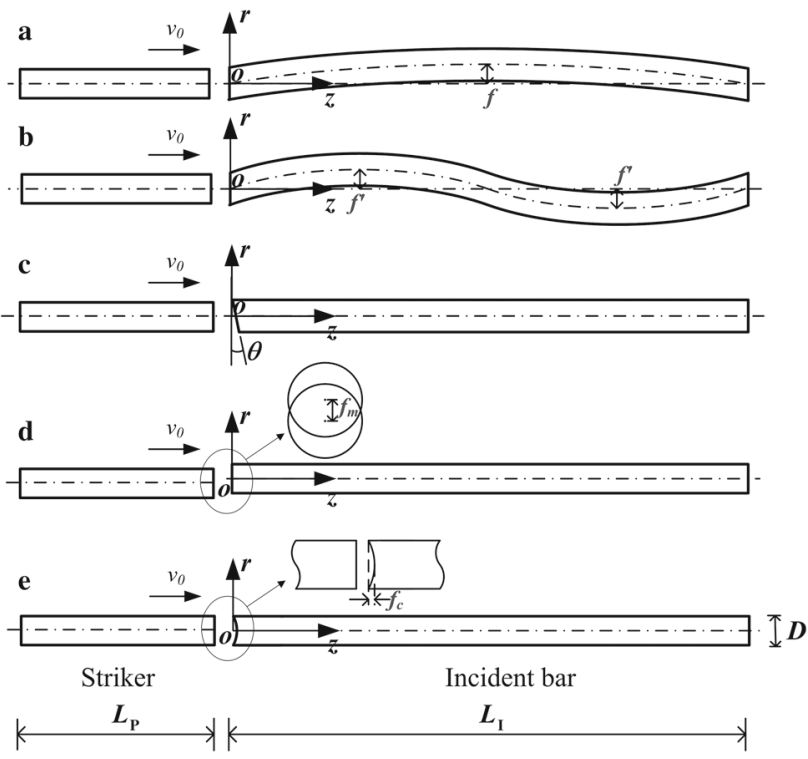

Fig. 2 Schematic of numerical simulation. Four kinds of imperfect experimental conditions were simulated. a, b Represent the condition of curved bars $(\mathrm{a}, \mathrm{b})$, in which deflections $f$ and $f^{\prime}$ represent the imperfect degree of the defects, respectively. c Represents the condition of inclination of the impact end-surface, in which $\theta$ denotes the contact angle. d Represents the condition of misalignment, in which $f_{\mathrm{m}}$ denotes the distance between the centers of the striker and the incident bar. e Represents the condition of indentation of the impact end-surface, in which $f_{\mathrm{c}}$ denotes the end-surface deflection

different angles of inclination $\beta$ were studied. Note that the inclination of the incident bar introduced two imperfect conditions into the experiments. One is the curved bar condition resulting in a change of propagation direction for stress waves, and another is the inclination of the impact endsurface leading to the change of the pulse shape because of impedance mismatch at the impact interface. The influences of the curved bar and the inclination of the impact end-surface were coupled in the experiments. To separate those effects, three-dimensional numerical models were designed as shown in Fig. 2. The defects of the curved bar were designed as shown in Fig. 2a and b, in which the impact end-surfaces were parallel to each other. The deflections $f$ and $f^{\prime}$ represented the degree of defects for curved bar (a) and curved bar (b), respectively. Again, in practical SHPB experiments, the defect of the curved bar $(a, b)$ are generally coupled with the defect of the inclination of the impact end-surface. Here, it was only numerically designed to decouple those effects. The experiment for the $\beta=0^{\circ}$ could be seen as the defect of the curved bar with an infinite radius of curvature. As shown in Fig. 2c, the contact angle of the incident bar, $\theta$, represents the degree of the defect for the inclination of the impact endsurface. Moreover, the effects of the misalignment between the striker and the incident bar are investigated. The degree of this defect is represented by the $f_{\mathrm{m}}$ as shown in Fig. $2 \mathrm{~d}$.
Table 1 Geometrical and material parameters of the SHPB system

\begin{tabular}{ll} 
Length of the striker $L_{\mathrm{p}}(\mathrm{cm})$ & 30 \\
Length of the incident bar $L_{\mathrm{T}}(\mathrm{cm})$ & 120 \\
Diameter of the striker $D_{\mathrm{p}}(\mathrm{mm})$ & 16 \\
Diameter of the incident bar $D_{\mathrm{I}}(\mathrm{mm})$ & 16 \\
Initial density $\rho\left(\mathrm{kg} / \mathrm{m}^{3}\right)$ & $7.85 \times 10^{3}$ \\
Young's modulus $E(\mathrm{GPa})$ & 206 \\
Poisson's ratio $v$ & 0.3 \\
Parameter $A(\mathrm{MPa})$ & 507 \\
Parameter $B(\mathrm{MPa})$ & 320 \\
Parameter $C$ & 0.064 \\
Parameter $n$ & 0.28 \\
\hline
\end{tabular}

Finally, the influence of the indentation of the impact endsurface is also simulated, and the degree of this defect is represented by the $f_{\mathrm{c}}$ as depicted in Fig. 2e. In simulation, the dimensions of the striker and the incident bar were the same as the experiments as listed in Table 1.

In simulation, the dimensions of the striker and the incident bar were the same as the experiments, as listed in Table 1 .

Using LS-DYNA software [26], an explicit nonlinear structural integration scheme was applied to analyze the dynamic response of the incident bar. The code has been validated to analyze the dynamic response such as in SHPB experiments [27,28]. The striker was meshed with 9216 eight-node brick elements, and the incident bar was meshed with 384000 eight-node brick elements. The striker and the incident bar were finely meshed with 768 elements at the cross-section. For the defects of the curved bar $(a, b)$, the elastic constitutive models was taken for both the striker and the incident bar. For other defects, the Johnson-Cook constitutive model without considering the effect of temperature [29] was taken for both the striker and the incident bar because plastically deformation maybe occurs near the impact region. The effective stress can be written as following,

$\sigma_{\mathrm{y}}=\left(A+B \bar{\varepsilon}^{\mathrm{p}^{n}}\right)\left(1+C \ln \dot{\varepsilon}^{*}\right)$,

where $\bar{\varepsilon}^{\mathrm{p}}$ is equivalent plastic strain, $\dot{\varepsilon}^{*}=\dot{\bar{\varepsilon}}^{\mathrm{p}} / \dot{\varepsilon}_{0}$ is the normalized equivalent plastic strain rate and $\dot{\varepsilon}_{0}=1 \mathrm{~s}^{-1}, A, B$, and $C$ are material constants, and $n$ is work hardening exponent. The material constitutive model related parameters are also given in Table 1 [23]. The weighted master-slave surface algorithm without considering friction effects [26] was chosen to simulate the impact condition between the striker and the incident bar.

\subsection{Validation of numerical modeling}

Firstly, the numerical model was validated with the PDV measurement results for $\beta=0^{\circ}, 1^{\circ}, 2^{\circ}$ (the PDV results will 


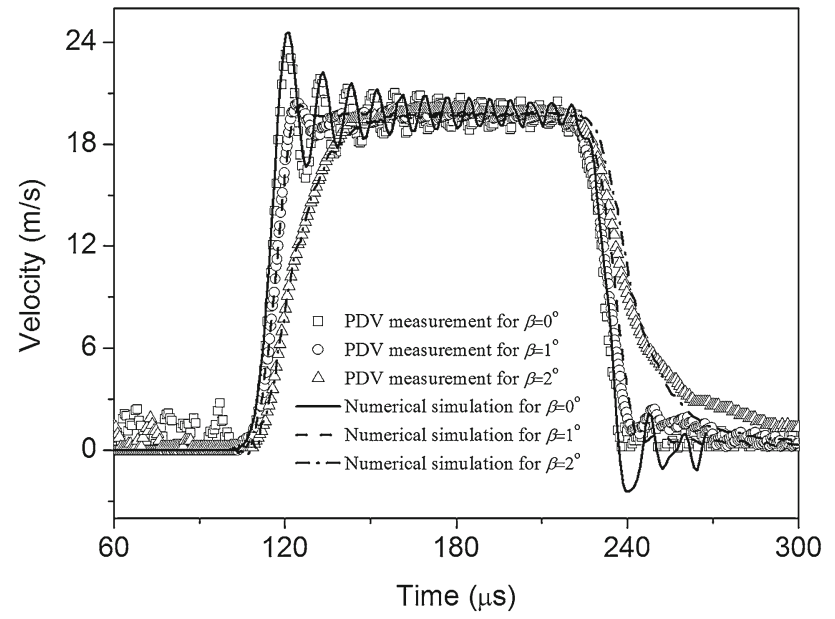

Fig. 3 Simulated longitudinal velocities and PDV-measured velocities at the end-surface's center of the incident bar. The velocity profiles were shifted to the same start time to make a clear comparison

be given in Sect. 4 as Fig. 5. Here it is just used to validate the numerical model). The simulated longitudinal velocities and the PDV measured velocities at the end-surface's center of the incident bar are given in Fig. 3, in which the velocity profiles are shifted to the same start time to make a clear comparison (the start time for the PDV measurement is the time that the stress waves arrive at the strain gauges and reaches the trigger value, whereas the start time for the numerical simulation is the start time to calculation).

The simulated end-surface velocities of the incident bar, including the rise time, the plateau, and the fall time are in good agreement with the PDV-measured results, except that the period of the oscillations induced by the lateral inertia effect is relative large in the numerical analysis for the inclination angle $\beta=0^{\circ}$. This may indicate that there are small differences in material parameters especially for Poisson's ratio used in the simulation when compared to the true values. Considering the dispersion effect induced by the lateral inertia, the stress wave speed $C$ with a wavelength $\lambda$ has the relation as follows,

$\frac{C}{C_{0}} \approx 1-v^{2} \pi^{2}\left(\frac{r_{0}}{\lambda}\right)^{2}$,

where $C_{0}$ is wave speed without considering the lateral inertia effect, $r_{0}$ is radius of the bar, $v$ is Poisson's ratio.

According to Eq. (2), for the same wavelength $\lambda$, the wave speed $C$ increases with decreasing Poisson's ratio, which implies that a slightly large Poisson's ratio is taken in the simulation. Nevertheless, the influence of the imperfect experimental conditions was well captured in the simulation as depicted in Fig. 3. In addition, the simulated velocities of the plateau and the pulse durations also agree with the experimental measurements, which show the consistency of the numerical model.

\section{Results and discussion}

\subsection{Experimental results}

The measured strain pulses and the end-surface velocities are shown in Figs. 4 and 5, respectively, in which the impact velocities of the striker measured by the laser velocimetry are $19.90 \mathrm{~m} / \mathrm{s}$ for $\beta=0^{\circ}, 19.84 \mathrm{~m} / \mathrm{s}$ for $\beta=1^{\circ}$, and 19.88 $\mathrm{m} / \mathrm{s}$ for $\beta=2^{\circ}$. The results as depicted in Figs. 4 and 5 reveal the dynamic responses of the incident bar, including the fast rising edge and the fluctuations at the plateau stage induced by the lateral inertia effect $[6,14,15,30-33]$. In addition, the end-surface velocities of the incident bar measured by the PDV system are about $19.80 \mathrm{~m} / \mathrm{s}$ on average, which is consistent with the laser velocimetry measurements. The

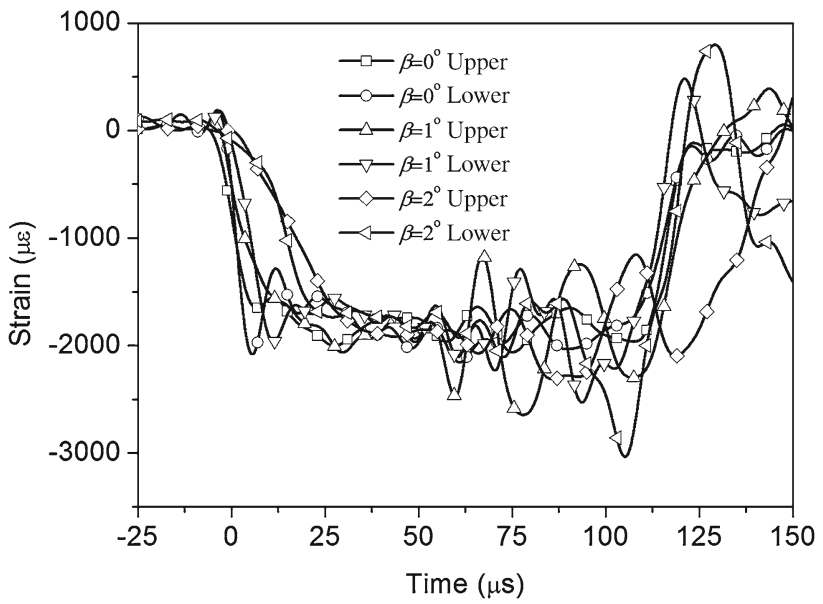

Fig. 4 Measured strain pulses at the upper and lower position at the middle of the incident bar. The impact velocities of the striker for the inclination angles $\beta=0^{\circ}, 1^{\circ}, 2^{\circ}$ are $19.90 \mathrm{~m} / \mathrm{s}, 19.84 \mathrm{~m} / \mathrm{s}, 19.88 \mathrm{~m} / \mathrm{s}$, respectively

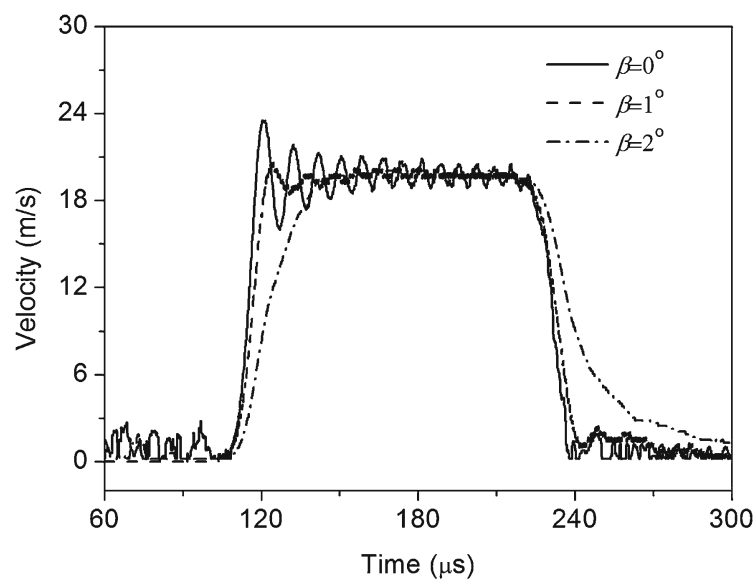

Fig. 5 Measured velocities at the center of the right end-surface of the incident bar. The impact velocities of the striker for the inclination angles $\beta=0^{\circ}, 1^{\circ}, 2^{\circ}$ are $19.90 \mathrm{~m} / \mathrm{s}, 19.84 \mathrm{~m} / \mathrm{s}$, and $19.88 \mathrm{~m} / \mathrm{s}$, respectively 
experimental results also capture the difference of the incident waves for the different inclination angles $\beta$. As shown in Fig. 4, the amplitudes of the bending waves and the rise time increase, and the amplitudes of the fluctuations decrease with an increasing inclination angle. The rise time also increases fast by increasing the angle $\beta$. The influence of the inclination angle on the incident waves of the incident bar is also captured clearly by the PDV system. As shown in Fig. 5, while increasing the inclination angle from $0^{\circ}$ to $2^{\circ}$, the rise time increases quickly and the fluctuation characteristics disappear gradually. Additionally, the fall time also increases with increasing the inclination angle, which is not obvious in the strain signals due to the bending waves. As the PDV measures the velocities at the center of the end-surface, the bending waves are not captured. Multi-point measurements of the PDV system are expected to be carried out to investigate the bending wave effects and the dispersion effects. According to the measurement results, it can be seen that the imperfect experimental conditions of the SHPB influence the incident waves significantly. Quantitative investigation for the imperfect conditions in SHPB experiments will be performed by numerical simulation in the next section.

\subsection{Simulation results and discussion}

\subsubsection{Effect of the curved bar}

Firstly, the numerically designed imperfect conditions of the curved bar $(\mathrm{a}, \mathrm{b})$ as depicted in Fig. 2a and b were simulated. Here, we take the longitudinal velocities $v_{z_{-} \text {Upper }}$ at the position $\left(r=D / 2, z=L_{\mathrm{I}} / 2\right)$ and $v_{z_{-} \text {Lower }}$ at the position $\left(r=-D / 2, z=L_{\mathrm{I}} / 2\right)$ to represent the dynamic response of the incident bar. The deflections $f$ were chosen as 6,8 , and $10 \mathrm{~mm}$ for the curved bar (a) and the deflections $f^{\prime}$ were designed as $2.5,3.5$, and 5.0 for the curved bar (b). The $v_{z_{-} \text {Upper }}$ for the different deflections $f$ are given in Fig. 6a, where $f=0.0 \mathrm{~mm}$ represents the perfect condition. The difference between the $v_{z_{-} \text {Upper }}$ and the $v_{z_{-} \text {Lower }}$, denoting the bending waves, are given in Fig. $6 b$. The simulated results were almost the same as each other for the various $f$ except a very slight increase of the bending waves' amplitude.

The $v_{z_{-} \text {Upper }}$ for the various $f^{\prime}$ are given in Fig. $7 \mathrm{a}$, where $f^{\prime}=0.0 \mathrm{~mm}$ represents the perfect condition. And the difference between the $v_{z_{-} \text {Upper }}$ and the $v_{z_{-} \text {Lower }}$, denoting the bending waves, for the various $f^{\prime}$ are given in Fig. 7b. Similar to the curved bar (a), the results of the curved bar (b) were also very close to each other, which implies that the incident waves will not be affected by the defect of the curved bar conditions.

In the simulation, the deflection $f=10.0 \mathrm{~mm}$ in the curved bar (a) and the $f^{\prime}=5.0 \mathrm{~mm}$ in the curved bar (b) were designed very large when compared to true experiments. However, the simulated dynamic responses of the
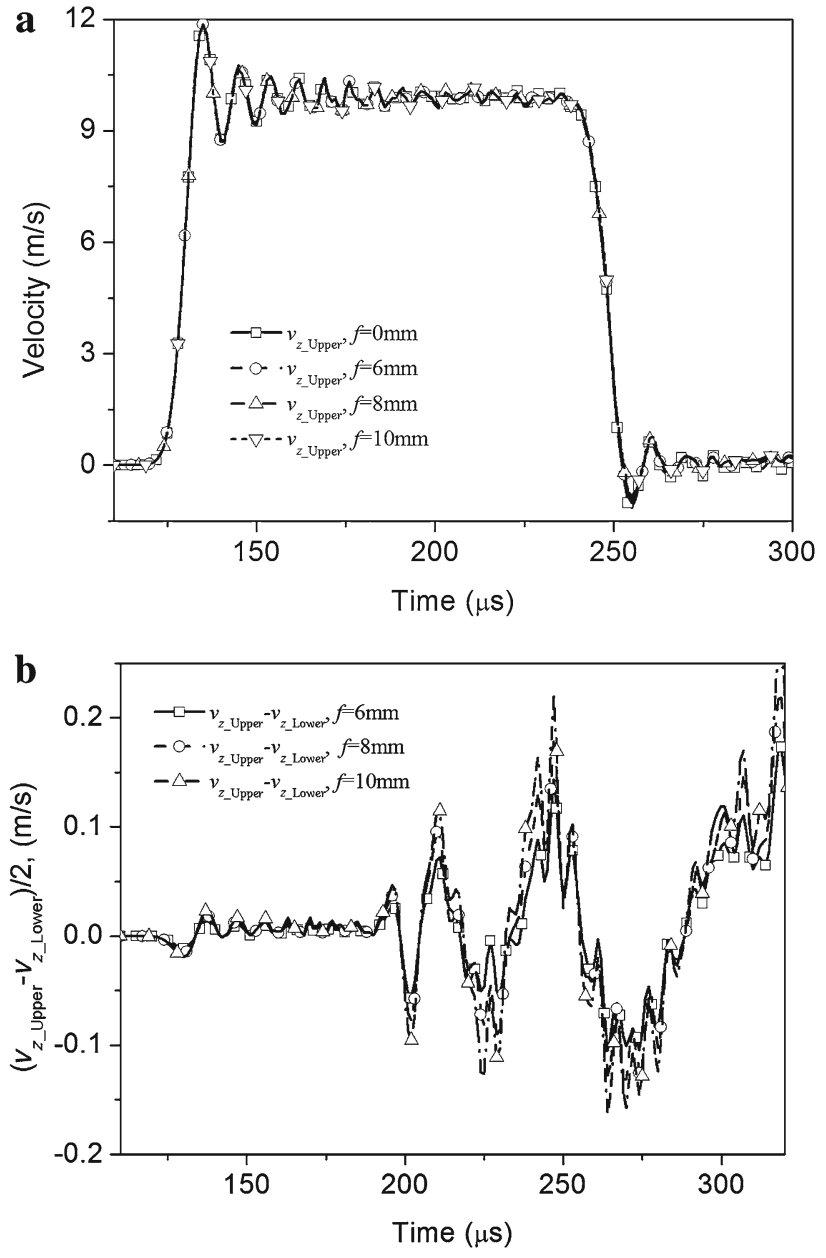

Fig. 6 a Velocities $v_{z_{-} \text {Upper }}$ and $v_{z_{-} \text {Lower }}$ for the deflections $f=6 \mathrm{~mm}$, $f=8 \mathrm{~mm}$ and $f=10 \mathrm{~mm}$ in the curved bar (a). The $f=0.0 \mathrm{~mm}$ represents the perfect condition. The curves are not discerned because they are closed to each other. b Difference between the $v_{z_{-} \text {Upper }}$ and the $v_{z_{-} \text {Lower }}$ denoting the bending waves

incident bar change very slightly under this condition. For the curved bar condition, the wavelength of the bar is much longer than that of the incident wave. As a result, the effects of the curvature on the stress waves are negligible while the stress waves propagate along the incident bar. Note that the curved bar condition generally leads to a non-parallel impact condition in practical SHPB experiments. Its effects should be carefully taken considered. In view of this, we only need to ensure the parallel impact end-surface condition regardless the curvature of bars in the SHPB.

\subsubsection{Effect of the inclination of the impact end-surface}

Nine contact angles $\theta=0.50^{\circ}, 0.75^{\circ}, 1.00^{\circ}, 1.25^{\circ}, 1.50^{\circ}$, $1.75^{\circ}, 2.00^{\circ}, 2.50^{\circ}$, and $3.00^{\circ}$ were simulated to investigate the influence of the inclination of the impact end-surface. The $v_{z_{-} \text {Upper }}$ for those contact angles are shown in Fig. 8. Since the bending wave speed is lower than the longitudinal wave 

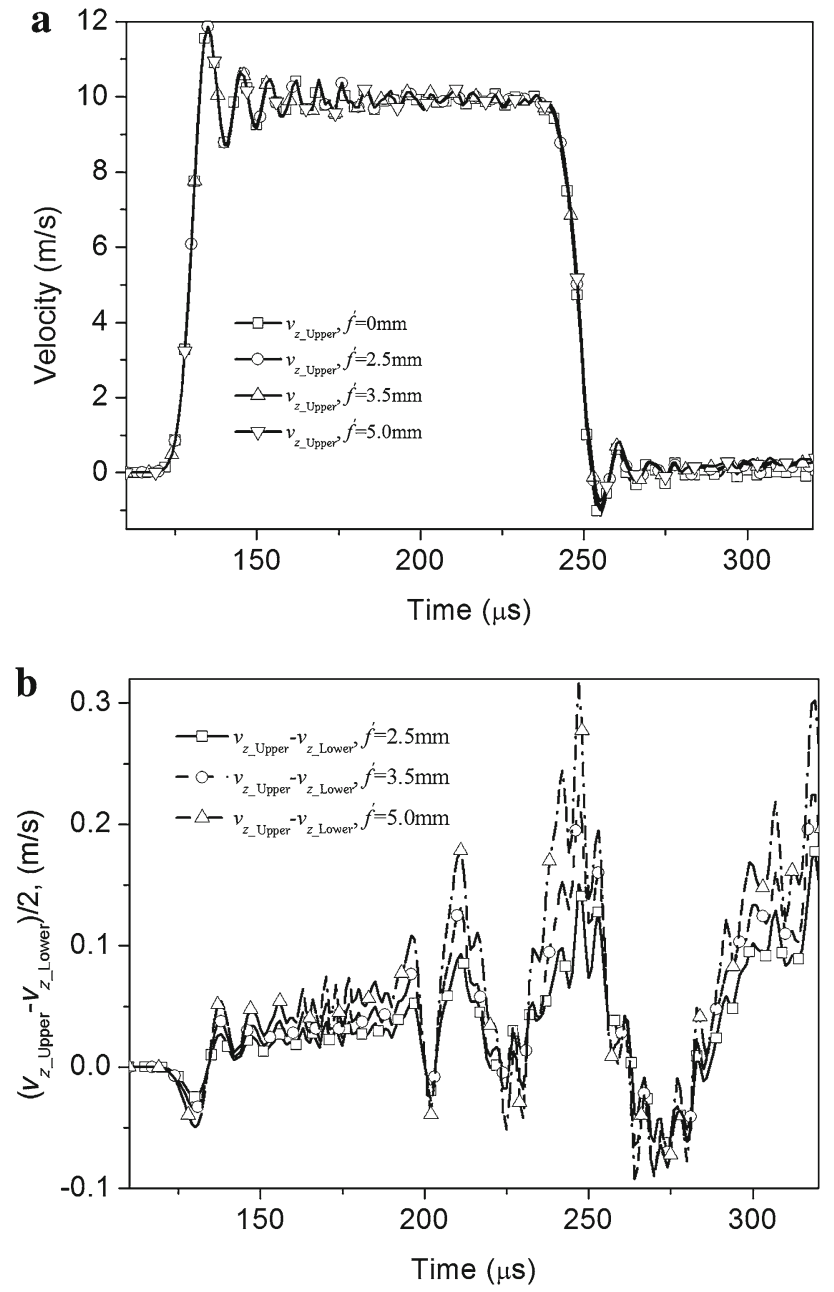

Fig. 7 a Velocities $v_{z \text { Upper }}$ and $v_{z \text { Lower }}$ for the deflections $f^{\prime}=$ $2.5 \mathrm{~mm}, f^{\prime}=3.5 \mathrm{~mm}$ and $f^{\prime}=5.0 \mathrm{~mm}$ in the curved bar (b). The $f=0.0 \mathrm{~mm}$ represents the perfect condition. The curves are not discerned because they are closed to each other. b Difference between the $v_{z_{-} \text {Upper }}$ and the $v_{z_{-} \text {Lower }}$ denoting the bending waves

speed, the bending waves do not affect the $v_{z_{-} \text {Upper }}$ at the early time. It can be seen that the $v_{z}$ Upper were significantly affected by the contact angles. With increasing the contact angle, the rise and the fall times increase rapidly, and the fluctuations at the plateau stage disappear gradually. A much ramped shape was obtained for a large impact angle. This might be of benefit for brittle materials such as concrete, ceramic, and cryogenic ice $[4,5,12,34-36]$, in which the rise time required is much longer than the time needed for the stress uniformity in the specimen. Figure 8 also shows that the amplitudes of the bending waves decrease gradually with an increasing impact angle. The region of plastic deformation increased with an increasing impact angle, which makes the stress uniform in the cross-section of the incident bar, and thereby decreases the amplitudes of the bending waves. The $v_{z \text { Lower }}$ for the different contact angle are shown in Fig. 9, which reveal the similar characteristics as the $v_{z_{-} \text {Upper }}$.

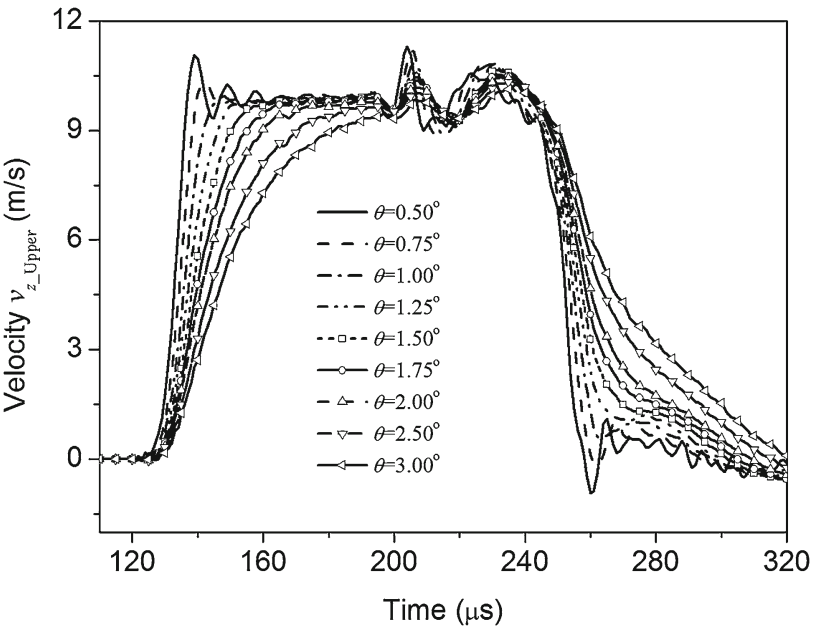

Fig. 8 Velocity $v_{z \text { Upper }}$ for the contact angles $\theta=$ $0.50^{\circ}, 0.75^{\circ}, 1.00^{\circ}, 1.25^{\circ}, 1.50^{\circ}, 1.75^{\circ}, 2.00^{\circ}, 2.50^{\circ}$, and $3.00^{\circ}$ in the inclination of the impact end-surface condition

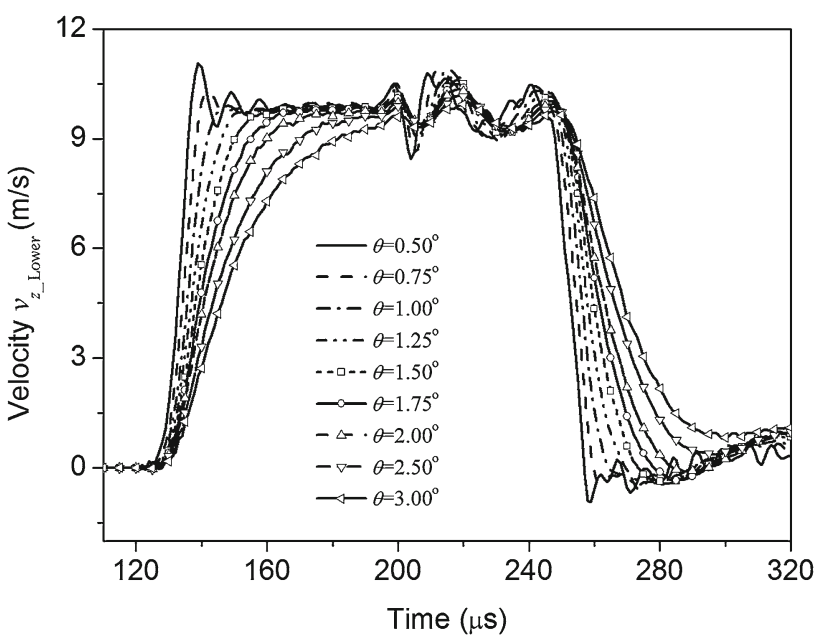

Fig. 9 Velocity $v_{z_{-} \text {Lower }}$ for the contact angles $\theta=$ $0.50^{\circ}, 0.75^{\circ}, 1.00^{\circ}, 1.25^{\circ}, 1.50^{\circ}, 1.75^{\circ}, 2.00^{\circ}, 2.50^{\circ}$, and $3.00^{\circ}$ in the inclination of the impact end-surface condition

Since the inclination of the impact end-surface acts as somewhat a kind of pulse shaper, the relationship between the rise time $\Delta t$ and the impact angle $\theta$ is depicted in Fig. 10. The rise time $\Delta t$ is determined as the duration for the velocity increasing from $0.3 \mathrm{~m} / \mathrm{s}$ (to ensure the precise selection of the start time) to $9.5 \mathrm{~m} / \mathrm{s}$ (to ensure all curves can reach this value) at the rising edge.

In view of Fig. 10, the rise time increases quickly at the beginning and then saturates gradually with increasing the impact angle. The rise time increased from about $9.03 \mu \mathrm{s}$ at $\theta=0.0^{\circ}$ to about $65.95 \mu \mathrm{s}$ at $\theta=3.0^{\circ}$. Note that the full width of the pulse is about $120 \mu \mathrm{s}$, which implies the rise time exceeds half of the full width for the impact angle $\theta=3.0^{\circ}$. The relationship between the rise time and the impact angle can be well fitted with a Boltzmann function with a correlation of $R^{2}=0.999$, 


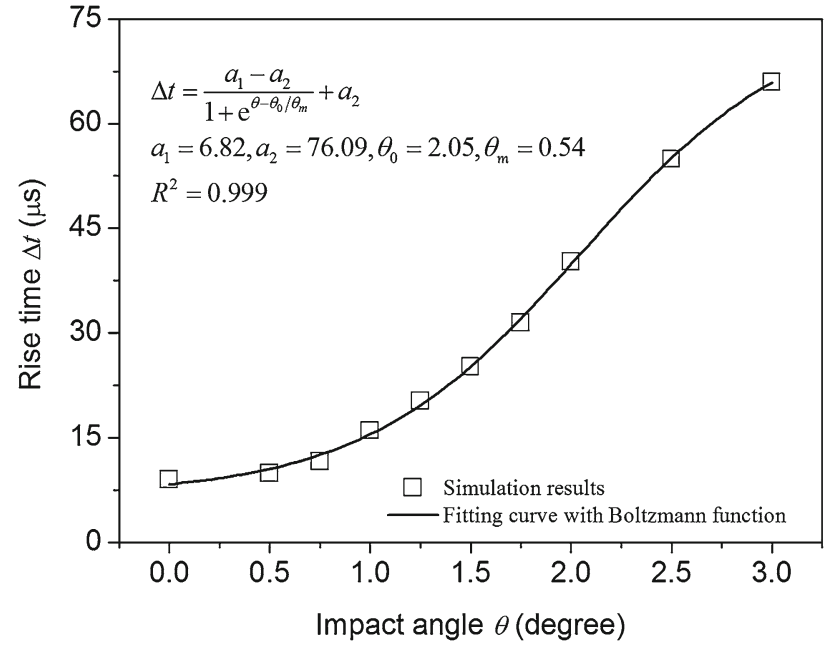

Fig. 10 Relationship between the rise time $\Delta t$ and the impact angle $\theta$. The rise time $\Delta t$ is determined as the duration for the velocity increasing from 0.3 to $9.5 \mathrm{~m} / \mathrm{s}$ at the rising edge

$\Delta t=76.09-\frac{69.27}{1+\mathrm{e}^{(\theta-2.05) / 0.54}}$.

Equation (3) is obtained only for the C1045 steel material. For other materials, there should be similar relationships.

In view of the simulated results of the curved bar and the inclination of the impact end-surface, the experimental results obtained in Sect. 2 were predominately affected by the defect of inclination of the impact end-surface. It is also obviously demonstrated in Fig. 11 (the curves are shifted to ensure the same start time), in which a comparison of velocities between the PDV measurements for the various $\beta$ and the simulated results for the various $\theta$ is given. The simulated results agree with the PDV measurements when $\theta=\beta$, indicating that the inclination of the impact end-surface is the predominant factor in the aforementioned SHPB experiments. Note that the incident stress waves at the defect of the inclination of the impact end-surface are accompanied by considerable bending waves, which are detrimental to the 1D stress wave assumption of SHPB experiments. The existence of the bending waves brings complications such as non-uniform stress distribution in a specimen. For this reason, in actual experiments the bending waves are required to be physically minimized or completely removed. Therefore, the inclination of the impact end-surface should be excluded from SHPB experiments except when only the rising edge is used.

\subsubsection{Effect of misalignment}

Eleven values $f_{\mathrm{m}} / D=0.01,0.02,0.04,0.06,0.08,0.10$, $0.12,0.14,0.16,0.18$, and 0.20 were simulated to investigate the influence of the misalignment between the striker and the incident bar on the dynamic response of the inci-

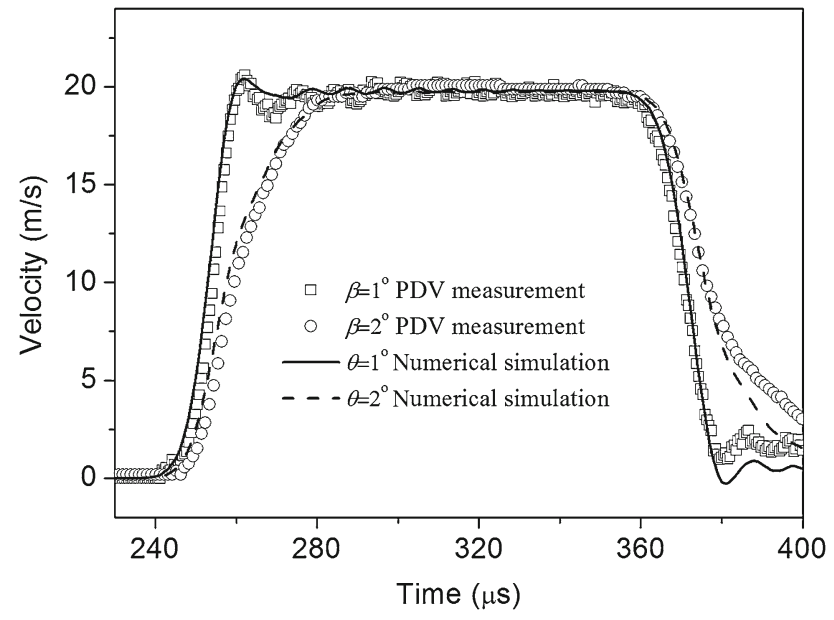

Fig. 11 Comparison of the incident bar's end-surface velocities at the center between the PDV measurements for various $\beta$ and the simulated results for various $\theta$. The curves are shifted to ensure the same start time

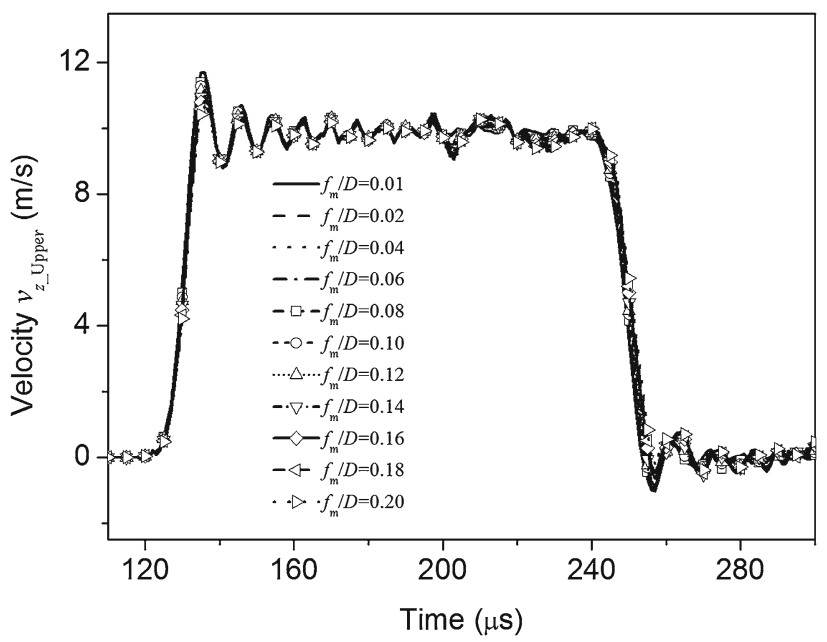

Fig. 12 Velocities $v_{z_{-} \text {Upper }}$ for the misalignment value $f_{\mathrm{m}} / D=$ $0.01,0.02,0.04,0.06,0.08,0.10,0.12,0.14,0.16,0.18$, and 0.20 in the misalignment condition

dent bar. The velocities $v_{z_{-} \text {Upper }}$ for the different $f_{\mathrm{m}} / D$ are shown in Fig. 12. Those velocity profiles are very close to each other except for the amplitudes of the fluctuations at the plateau and the bending waves. Also, the amplitudes of the fluctuations decrease with an increasing misalignment value $f_{\mathrm{m}} / D$, which might be ascribed to the shear deformations that required retaining a vertical cross-section. Moreover, the bending waves are generated under this condition. The amplitudes of the bending waves increase with an increasing misalignment value $f_{\mathrm{m}} / D$, which is ascribed to the increase of bending moment the cross-section.

To show clearly the effects of the misalignment, $v_{z_{-} \text {Upper }}$ and $v_{z_{\text {LL Lower }}}$ for $f_{\mathrm{m}} / D=0.01,0.06,0.12$, and 0.18 are given in Fig. 13. From Fig. 13, the velocities $v_{z_{-} \text {Upper }}$ and $v_{z_{-} \text {Lower }}$ are almost the same as each other except after the 


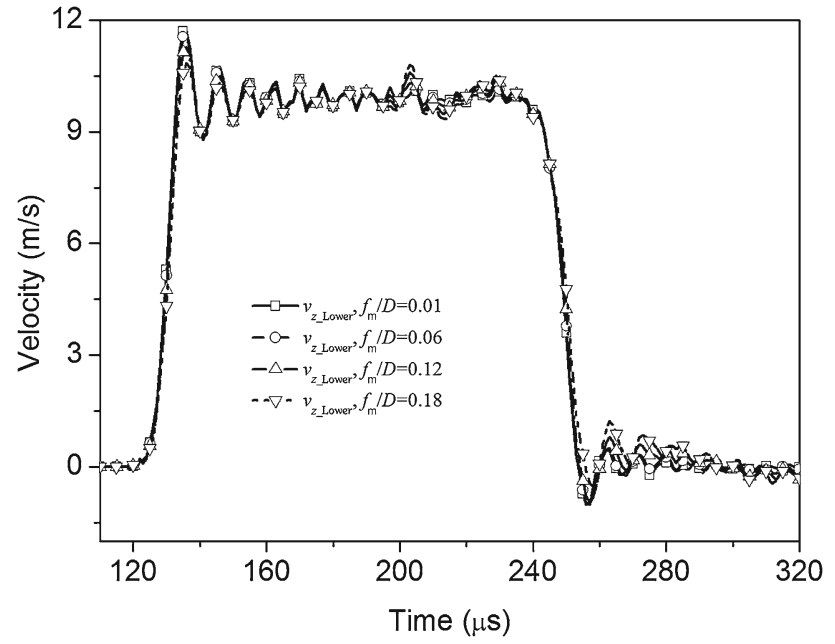

Fig. 13 Velocities $v_{z \text { Lower }}$ for the misalignment value $f_{\mathrm{m}} / D=$ $0.01,0.06,0.12$, and 0.18 in the misalignment condition

bending waves arrive. Unlike the inclination of the impact end-surface condition, the rise and the fall times are nearly not changed by the misalignment value $f_{\mathrm{m}} / D$. In addition, the amplitudes of the bending waves are relatively lower when compared to that obtained under the inclination of the impact end-surface condition. However, similar to the inclination of the impact end-surface, the specimen will be loaded non-uniformly because of the existence of the bending waves, which should be excluded from SHPB experiments.

\subsubsection{Effect of the indentation of the impact end-surface}

Finally, eleven surface deflections $f_{\mathrm{c}} / D=0.05,0.1,0.2$, $0.3,0.4,0.5,0.6,0.7,0.8,0.9$, and 1.0 were simulated to investigate the influence of the indentation of the impact end-surface. The simulated velocities $v_{z_{-} \text {Upper }}$ for the different $f_{\mathrm{c}} / D$ are given in Fig. 14, in which the insert is the radial distribution of the $v_{z_{-} \text {Upper }}$ for $f_{\mathrm{c}} / D=1.0$. From Fig. 14, the $v_{z_{-} \text {Upper }}$ are significantly affected by the indentation of the impact end-surface condition. By increasing the impact end-surface deflection $f_{\mathrm{c}} / D$, the rise and the fall times increased quickly, and the fluctuations disappear gradually. A much ramped pulse shape was obtained for a large impact end-surface deflection. In addition, as the geometry of the incident bar was symmetrical for the designed impact end-surface deflection condition, the bending waves were not generated. As shown in the insert in Fig. 14, the velocities along the cross-section were uniform. The region of plastic deformation increased with increasing the impact end-surface deflection, which makes the stress uniform in the cross-section.

Similar to the inclination of the impact end-surface, the indentation of the impact end-surface also acts somewhat as a kind of pulse shaper. The relationship between the rise time

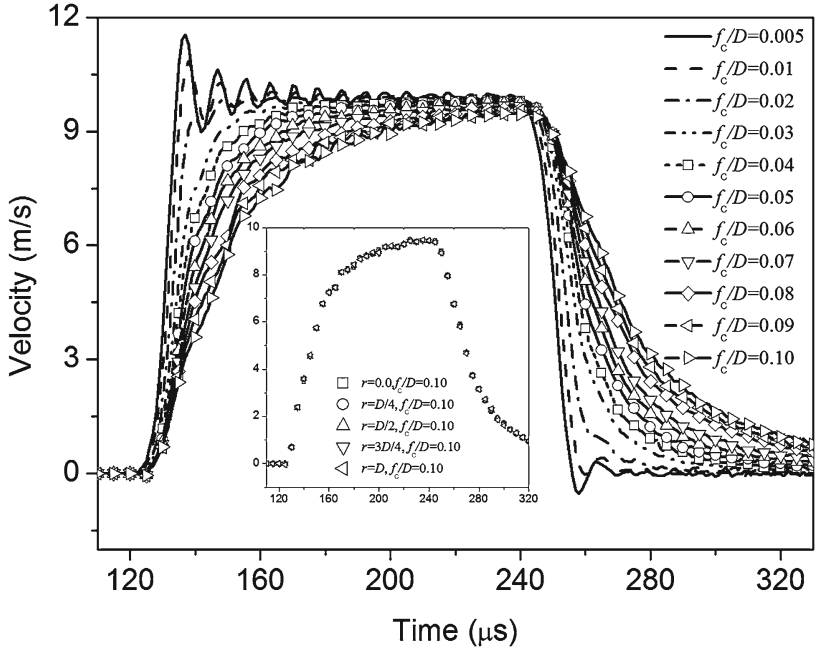

Fig. 14 Velocities $v_{z_{-} \text {Upper }}$ for the surface deflections $f_{\mathrm{c}} / D=$ $0.05,0.1,0.2,0.3,0.4,0.5,0.6,0.7,0.8,0.9$, and 1.0 in the indentation of the impact end-surface condition. The insert is the radial distribution of $v_{z_{-}}$Upper for $f_{\mathrm{c}} / D=1.0$

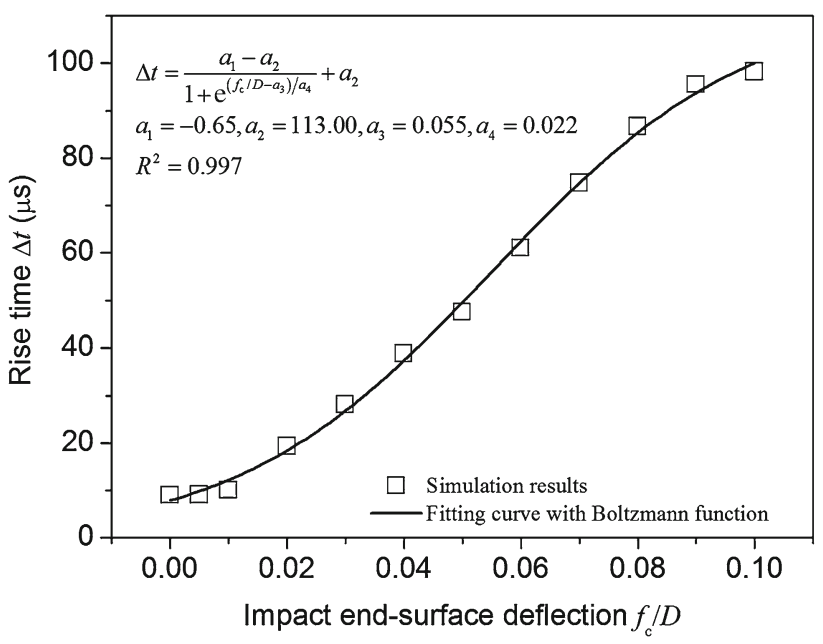

Fig. 15 Relationship between the rise time $\Delta t$ and the impact endsurface deflection $f_{\mathrm{c}} / D$. The rise time $\Delta t$ is determined as the duration for the velocity increasing from $0.3 \mathrm{~m} / \mathrm{s}$ to $9.5 \mathrm{~m} / \mathrm{s}$ at the rising edge

$\Delta t$ and the impact end-surface deflection $f_{\mathrm{c}} / D$ is depicted in Fig. 15. The rise time $\Delta t$ is also determined as the duration for velocity increasing from $0.3 \mathrm{~m} / \mathrm{s}$ (to ensure the precise selection of the start time) to $9.5 \mathrm{~m} / \mathrm{s}$ (to ensure all curves can reach this value) at the rising edge.

In view of Fig. 15, the rise time increases quickly at the beginning and then saturates gradually with increasing $f_{\mathrm{c}} / D$. The rise time increases from about $9.03 \mu \mathrm{s}$ for $f_{\mathrm{c}} / D=0.0$ to about $98.19 \mu \mathrm{s}$ for $f_{\mathrm{c}} / D=0.1$. The relationship between the rise time and the impact end-surface deflection can be well fitted with a Boltzmann function with a correlation of $R^{2}=0.997$,

$\Delta t=113.00-\frac{113.65}{1+\mathrm{e}^{\left(f_{\mathrm{c}} / D-0.055\right) / 0.022}}$. 
Again, Eq. (4) is obtained only for the C1045 steel material. For other material, there should be a similar relationship.

\section{Conclusions}

In this paper, the effects of the imperfect experimental conditions on the incident waves of SHPB experiments were investigated by experiments and numerical simulations. The main conclusions are as follows:

(1) The incident waves of SHPB experiments change significantly while changing the inclination angle of the incident bar. The rise and fall times increase quickly, and the fluctuations at the plateau stage disappear gradually by increasing the inclination angle.

(2) Since the wavelength of a curved bar is much longer when compared to the wavelength of the incident waves, the effects of the curved bar are negligible. Nevertheless, the curved bar condition is usually coupled with the inclination of the impact end-surface condition in experiments.

(3) The inclination of the impact end-surface influences the incident waves significantly. With increasing the contact angle, the pulse becomes much ramped, and the fluctuations at the plateau stage disappear gradually. The bending waves are generated at this condition, and the amplitude of the bending wave decreases by increasing the impact angle.

(4) The misalignment between the striker and the incident bar influences the fluctuations at the plateau stage and the bending waves modestly. The amplitudes of the fluctuations decrease and the bending waves increase by increasing the misalignment value $f_{\mathrm{m}} / D$.

(5) The indentation of the impact end-surface has great impact on the incident waves. The rise and fall times increase, and the fluctuations at the plateau stage disappear gradually by increasing the impact end-surface deflection $f_{\mathrm{c}} / D$.

(6) Both of the defects of inclination of the impact endsurface and the indentation of the impact end-surface act somewhat as a kind of pulse shaper. The rise time increases by increasing the impact angle or by increasing the impact end-surface deflection.

Acknowledgments The authors would like to thank the National Natural Science Foundation of China (Grants 11402277 and 11332011) for financial support.

\section{References}

1. Hopkinson, B.: A method of measuring the pressure produced in the detonation of high explosives or by the impact of bullets. Philos. Trans. R. Soc. Lond. A 213, 437-456 (1914)
2. Kolsky, H.: An investigation of the mechanical properties of materials at very high rates of loading. Proc. R. Soc. Lond. B 62, 676-700 (1949)

3. Davies, R.: A critical study of the Hopkinson pressure bar. Philos. Trans. R. Soc. London A 240, 375-457 (1948)

4. Chen, W.N.W., Song, B.: Split Hopkinson (Kolsky) Bar: Design, Testing and Applications. Springer, New York (2011)

5. Zhu, J., Hu, S., Wang, L.: An analysis of stress uniformity for concrete-like specimens during SHPB tests. Int. J. Impact Eng. 36, 61-72 (2009)

6. Meng, H., Li, Q.: Correlation between the accuracy of a SHPB test and the stress uniformity based on numerical experiments. Int. J. Impact Eng. 28, 537-555 (2003)

7. Nemat-Nasser, S.: Introduction to high strain rate testing, ASM Handbook, ASM International, OH, pp. 427-428 (2000)

8. Nemat-Nasser, S., Isaacs, J.: Direct measurement of isothermal flow stress of metals at elevated temperatures and high strain rates with application to Ta and TaW alloys. Acta Mater. 45, 907-919 (1997)

9. Zhao, H., Abdennadher, S., Othman, R.: An experimental study of square tube crushing under impact loading using a modified large scale SHPB. Int. J. Impact Eng. 32, 1174-1189 (2006)

10. Bailly, P., Delvare, F., Vial, J., et al.: Dynamic behavior of an aggregate material at simultaneous high pressure and strain rate: SHPB triaxial tests. Int. J. Impact Eng. 38, 73-84 (2011)

11. Li, Y., Guo, Y., Hu, H., et al.: A critical assessment of hightemperature dynamic mechanical testing of metals. Int. J. Impact Eng. 36, 177-184 (2009)

12. Shazly, M., Prakash, V., Lerch, B.A.: High strain-rate behavior of ice under uniaxial compression. Int. J. Solids Struct. 46, 1499-1515 (2009)

13. Yang, L., Shim, V.: An analysis of stress uniformity in split Hopkinson bar test specimens. Int. J. Impact Eng. 31, 129-150 (2005)

14. Zhao, H.: Material behaviour characterisation using SHPB techniques, tests and simulations. Comput. Struct. 81, 1301-1310 (2003)

15. Merle, R., Zhao, H.: On the errors associated with the use of large diameter SHPB, correction for radially non-uniform distribution of stress and particle velocity in SHPB testing. Int. J. Impact Eng. 32, 1964-1980 (2006)

16. Lifshitz, J., Leber, H.: Data processing in the split Hopkinson pressure bar tests. Int. J. Impact Eng. 15, 723-733 (1994)

17. Follansbee, P., Frantz, C.: Wave propagation in the split Hopkinson pressure bar. J. Eng. Mater. Tech. 105, 61-66 (1983)

18. Yew, E., Chen, C.: Experimental study of dispersive waves in beam and rod using FFT. J. Appl. Mech. 45, 940-943 (1978)

19. Song, L., Hu, S.: Correction of end-face indentation in SHPB test. Explo. Shock Wave 30, 0203-0208 (2010)

20. Dolan, D.H.: Accuracy and precision in photonic Doppler velocimetry. Rev. Sci. Instrum. 81, 053905-053912 (2010)

21. Dolan, D.H., Jones, S.C.: Push-pull analysis of photonic Doppler velocimetry measurements. Rev. Sci. Instrum. 78, 076102-076105 (2007)

22. Strand, O.T., Goosman, D.R., Martinez, C., et al.: Compact system for high-speed velocimetry using heterodyne techniques. Rev. Sci. Instrum. 77, 083108-083116 (2006)

23. Chen, G., Chen, Z., Tao, J., et al.: Investigation and validation on plastic constitutive parameters of 45 steel. Explos. Shock Waves 25, 451-456 (2005)

24. Wu, X., Duan, Z., Song, H., et al.: Shock pressure induced by glass-confined laser shock peening: experiments, modeling and simulation. J. Appl. Phys. 110, 053112 (2011)

25. Song, H., Wu, X., Huang, C., et al.: Measurement of fast-changing low velocities by photonic Doppler velocimetry. Rev. Sci. Instrum. 83, 073301 (2012) 
26. Manual, L-DKUs.: Version 970, Livermore Software Technology Corporation 1-46 (2003)

27. Moura, R.T., Clausen, A.H., Fagerholt, E., et al.: Impact on HDPE and PVC plates-experimental tests and numerical simulations. Int. J. Impact Eng. 37, 580-598 (2010)

28. Tasdemirci, A., Hall, I.W.: Numerical and experimental studies of damage generation in a polymer composite material at high strain rates. Polym. Test. 25, 797-806 (2006)

29. Johnson, G., Cook, W.: A constitutive model and data for metals subjected to large strains, high strain rates and high temperatures, In: Proceedings of the 7th International Symposium on Ballistics, pp. 541-547. The Hague, The Netherlands (1983)

30. Aleyaasin, M., Harrigan, J.J.: Wave dispersion and attenuation in viscoelastic polymeric bars: analysing the effect of lateral inertia. Int. J. Mech. Sci. 52, 754-757 (2010)

31. Field, J.E., Walley, S.M., Proud, W.G., et al.: Review of experimental techniques for high rate deformation and shock studies. Int. J. Impact Eng. 30, 725-775(2004)
32. Joo Kim, D., Sirijaroonchai, K., El-Tawil, S., et al.: Numerical simulation of the split Hopkinson pressure bar test technique for concrete under compression. Int. J. Impact Eng. 37, 141-149 (2010)

33. Zhao, H., Elnasri, I., Abdennadher, S.: An experimental study on the behaviour under impact loading of metallic cellular materials. Int. J. Mech. Sci. 47, 757-774 (2005)

34. Song, B., Chen, W.: Split Hopkinson pressure bar techniques for characterizing soft materials. Lat. Am. J. Solids Struct. 2, 113-152 (2005)

35. Christensen, R., Swanson, S., Brown, W.: Split-Hopkinson-bar tests on rock under confining pressure. ExM 12, 508-513 (1972)

36. Li, X., Liu, D., Zhao, P.: Testing and response of large diameter brittle materials subjected to high strain rate. J. Mater. Civ. Eng. 14, $262(2002)$ 